\title{
Concepto de coeducación en el profesorado de Educación Física y metodología utilizada para su trabajo.
}

\author{
Pedro Angel Valdivia-Moral \\ Miriam López-López** \\ Amador Jesús Lara-Sánchez ${ }^{* * *}$ \\ Maria Luisa Zagalaz-Sánchez.***
}

\begin{abstract}
Resumen: El objetivo de esta investigación fue conocer cual es la concepción general del profesorado de Educación Física (EF) sobre la coeducación en EF y que estrategias metodológicas utiliza el profesorado para trabajarla. Se realizaron entrevistas semiestructuradas sometidas a análisis de contenido. Se destaca que; la categoría "Definición de coeducación" no existe unanimidad en el profesorado sobre que es la coeducación. La categoría "Utilización del espacio motriz" pone de manifiesto que los chicos utilizan la mayoría del espacio. La categoría "Lenguaje", resalta que el profesorado admite que existe un lenguaje sexista en los centros escolares pero que su uso es inconsciente.
\end{abstract}

Palabras clave: Educación Física. Metodología. Enseñanza

\section{INTRODUCCION}

La coeducación que tiene una importancia capital en la formación personal del alumnado, en el área de EF, donde se ponen de manifiesto las diferencias fisiológicas de ambos sexos cobra aun

\footnotetext{
'Departamento de Didáctica de la Educación Física, Plástica y Musical. Facultad de Educación. Universidad de Cádiz, Cádiz, España. E-mail: pedroangel.valdivia@uca.es

"Departamento de Nutrición y Bromatología. Facultad de Farmacia. Universidad de Granada. Granada, España. E-mail:miriloplop@hotmail.com

"'Departamento de Didáctica de la Expresión Musical, Plástica y Corporal. Universidad de Jaén. Universidad de Jaén, Jaén, España. E-mail: alara@ujaen.es

"...'Departamento de Didáctica de la Expresión Musical, Plástica y Corporal. Universidad de Jaén. Universidad de Jaén, Jaén, España. E-mail: Izagalaz@ujaen.es
} 
mayor importancia. Esto conlleva, que las chicas sean consideradas un problema desde el punto de vista del rendimiento deportivo (AZZARITO; SOLMON, 2009), y por eso, aunque hay voces disonantes en este sentido, es fundamental que el profesorado de EF conozca realmente que es la coeducación para poder trabajar con una metodología adecuada.

Existe controversia sobre el significado de coeducación, siendo diferente en función del área de conocimiento que se consulte. En el campo de la EF no existe unanimidad en definirla y, en muchas ocasiones se confunde con "igualdad de género". La diferencia entre ambos términos es clara para Zagalaz et al. (2000), cuando afirman que la coeducación, debe promover la igualdad de trato entre sexos, no aceptando el modelo universal masculino y desarrollando las cualidades de cada individuo. Como se puede comprobar la igualdad es necesaria para poder desarrollar la coeducación, sin embargo, creemos que la coeducación debe tener en cuenta que se trabaja con dos sexos diferentes que conviven en una realidad social común. Por otro lado, son varios los autores (CASTILLO; MARTÍNEZLÓPEZ; ZAGALAZ, 2010; SUBIRATS; BRULLET, 1988; VÁZQUEZ, 1990) que identifican las diferencias entre educación mixta y coeducación, no aceptando que la educación mixta conseguida con la Ley General de 1970 aporte consigo la coeducación.

El profesorado debe buscar el desarrollo integral e individual de niños y niñas. En este sentido, Lillo, Brotons y Simón (2006) entienden la coeducación como un sistema pedagógico integral que busca el desarrollo del alumnado en condiciones de libertad, equidad y correspondencia.

Por otro lado, Martínez-Galindo (2006) indica que coeducar significa enseñar a valorar, tanto a niños como a niñas, los rasgos positivos y negativos de los modelos masculino y femenino. Esta autora, prosigue indicando que se trata de hacer participe a los chicos de las formas de conducta que hasta ahora han sido consideradas femeninas, es decir, valorar las formas de comportamientos femeninas 
de igual manera que las masculinas. Por su parte, Valdivia, Sánchez, Alonso y Zagalaz (2011), añaden que la coeducación debe tener en cuenta aspectos como la afectividad, emociones y los valores morales.

Hasta ahora, hemos analizado aquellos enfoques sobre coeducación que se centran en las diferencias entre sexos y la valoración de las características de ambos sexos así como las características personales. Sin embargo, existen autores como Bonal (1997), Freixas et al. (1993) y Cervelló et al. (2003), que se centran en los recursos y en la igualdad en el proceso educativo.

Tal y como se ha puesto de manifiesto existen diferentes concepciones sobre el papel de la coeducación en EF. Por tanto, es necesario conocer que entiende el profesorado de secundaria de EF sobre que significa la coeducación.

Una vez conocidas cuales son las principales ideas sobre coeducación en EF que defienden los autores, es importante conocer como se trabaja. La importancia del análisis de cual es la metodología de trabajo utilizada por parte del profesorado, radica en que tal y como señalan Soler (2006) y Vázquez, Fernández-García y Ferro (2000) el comportamiento del alumnado y las creencias del profesorado siguen siendo muy estereotipadas, generándose en múltiples ocasiones situaciones de desigualdad. Estas situaciones se dan en la distribución del espacio y el material, las interacciones entre chicos y chicas, y el tipo de estrategias didácticas que utilizan los docentes. Estas situaciones se desarrollan desde una perspectiva de prejuicio hacia las niñas, que sin embargo, también han privado a los niños a participar en diferentes actividades "propias de niñas". En España, son varios los estudios que demuestran que el profesorado aún mantiene prejuicios sexistas que pueden incidir en la formación del alumnado (BONAL, 1997; SOLER, 2006; PIEDRA; GARCÍAPÉREZ; REBOLLO; RIES, 2011; VALDIVIA, 2012). Por ello, el efecto que tiene el profesorado sobre el alumnado en este aspecto ha sido estudiado en el contexto español por Bolaños y Jiménez (2007), Castillo y Corral (2011) y Castillo, Romero, González y Campos (2012). 
Asimismo, son varios los elementos educativos que intervienen en el proceso de enseñanza-aprendizaje en las clases de EF desde una perspectiva coeducadora. Nos referimos a los bloques de contenidos (VALDIVIA;SÁNCHEZ;ALONSO; ZAGALAZ, 2011), utilización del espacio motriz, las actividades en clase de EF, el tipo de agrupaciones utilizadas por el profesorado, el material, el lenguaje, la indumentaria, la evaluación y el feedback que lleva a cabo el profesorado. No obstante, y debido a que cada uno de estos factores necesita un análisis pormenorizado, nos centraremos en la utilización del espacio motriz, el tipo de agrupaciones, lenguaje y el material utilizado por tratar aspectos que inciden durante el desarrollo de la sesión de EF.

Ante esta situación, el objetivo de esta investigación no puede ser otro que conocer cual es la concepción general del profesorado de EF sobre la coeducación en EF y que estrategias metodológicas utiliza el profesorado para trabajarla.

\section{Método}

\subsection{Participantes}

La población de estudio estuvo compuesta por 9 profesores de EF que impartían docencia a alumnado entre 12 y 16 años perteneciente a la etapa de Educación Secundaria Obligatoria (ESO), tanto en centros públicos como en centros concertados de diferentes provincias españolas. Esta etapa corresponde a los cursos de $7^{\circ}, 8^{\circ}$ y $9^{\circ}$ curso de la enseñanza fundamental y los cursos de $1^{\circ}$ y $2^{\circ}$ de enseñanza media, del sistema educativo brasileño. Los participantes eran 4 mujeres y 5 hombres, con edades entre 35 y 57 años. Dichos profesores y profesoras habían ocupado diferentes puestos directivos a los largo de su experiencia. El muestreo que se utilizo fue no probabilístico de casos típicos (HEINEMANN, 2008). De esta manera, la experiencia en docencia de la EF menor a diez años fue un factor de exclusión. Dentro de las características cualitativas de este estudio, se utilizó un enfoque interpretativo y descriptivo en el 
que se optó por la calidad de la información recibida en las entrevistas, y no siendo el objetivo del muestreo la generalización de los resultados (RUÍZ; CAMPS, 2009; VALDIVIA et al., 2011).

\subsection{Procedimiento}

La primera fase de esta investigación fue la elaboración del marco teórico en el que se realizó una profunda revisión de la literatura científica sobre la materia. Así, se identificó cual era el estado de la cuestión y la necesidad de abordar un trabajo de este tipo.

La siguiente fase se subdivide en tres. En la primera, se fijan los objetivos de la investigación, de tal manera que se posibilita llevar a cabo una selección de la información necesaria para el guion de la entrevista. La siguiente fase trató la formación y entrenamiento de los investigadores en el tratamiento de las entrevistas, en función de la propuesta de Anguera (2003). Para ello, se utilizaron técnicas de "role playing", con el objetivo de asimilar las capacidades necesarias para llevar a cabo las entrevistas. Por último, se elaboró y validó la entrevista utilizando las pautas de Campoy (2006), tales como; a) creación de preguntas de tipo semiestructurado y de enfoque cualitativo; b) realización de un estudio piloto mediante dos entrevistas a licenciados en Ciencias de la Actividad Física y del Deporte (CAFD); c) consulta a jueces expertos para dar validez interna al instrumento. Dichos jueces expertos eran Doctores en CAFD, Filosofía y Pedagogía. El objetivo de esta fase fue la corrección del sesgo y obtener la versión definitiva de la entrevista.

En la tercera fase del procedimiento se contactó con la población de estudio con el objetivo de concertar las fechas de las entrevistas y mandarles el consentimiento informado que posteriormente se recogía. Una vez realizadas las entrevistas, se procedió a la recogida de los datos cualitativos procedentes de la transcripción de los discursos del profesorado.

Por último, cabe destacar que en este estudio y siguiendo las recomendaciones de Mosquera y Puig (1998) y Pérez y Carbó (2010), se utilizará el término "sexo" cuando nos referimos a diferencias 
biológicas. Por otro lado, se recurrirá al término "género" cuando se haga mención a la forma en las que las diferencias biológicas se interpretan en valores, expectativas sociales, etc., de cada uno de los sexos.

\subsection{INSTRUMENTO Y MATERIALES}

Tal y como indica Corbetta (2007), el seguimiento del guión de la entrevista por parte del investigador dio la posibilidad a que la manera de formular las preguntas quede en función de la valoración del entrevistador, posibilitándose que tal y como indica Álvarez-Gayou (2006), se profundizará en las experiencias del profesorado con el objetivo de buscar un enfoque cualitativo en la investigación.

En cuanto a las características de la entrevista, se siguieron los pasos descritos en Valdivia et al. (2011) para obtener los diferentes tipos de fiabilidad característicos en este tipo de instrumentos.

\subsection{ANÁLISIS DE CONTENIDO}

Para poder interpretar el sentido de los discursos de las entrevistas se sometieron a análisis de contenido los textos transcritos. Para ello, se utilizó el modelo de análisis cualitativo de textos adaptados de Heinemann (2008). De esta manera, en primer lugar se seleccionaron y organizaron los datos en función del sistema de categorías a priori, para posteriormente realizar el análisis de datos. Por lo tanto, existían preguntas en las que su respuesta daba lugar a la creación de nuevas subcategorías y/o indicadores.

\section{RESUlTAdOS Y discusión}

\subsection{Categoría Definición de Coeducación}

En esta categoría se analiza las diferentes versiones que tiene el profesorado de EF de secundaria sobre el concepto de coeducación, destacando aquellas opiniones que coinciden con las definiciones aportadas por los diferentes autores. 
En primer lugar encontramos las definiciones de coeducación con principal alusión a recursos y ámbito. Bonal (1997) indica que conseguir la coeducación es controlar que los recursos educativos se ofrezcan equilibradamente a cada grupo sexual y no hagan diferencias entre niños y niñas. En esta línea, encontramos en las entrevistas:

[...] la coeducación es la educación juntos, juntos referidos ¿A qué?, puede ser con alumnos de educación especial e integración en las aulas [...] hablando de contextos va referido a la distinción, agrupación de colegios, de alumnos, por sexo, un solo género [...] la terminología no va implícita al género sino más bien al contexto (\#2)(1).

Por otro lado, encontramos aquellas definiciones en las que la concepción de coeducación con un enfoque más dedicado al proceso educativo. Fontecha (2006), opina que coeducar es asegurar un trato equitativo para chicas y chicos, superando los estereotipos de género al llevar a la práctica cada una de las actividades que se desarrollan en el ámbito educativo. En este sentido, en las entrevistas se recoge las siguientes opiniones "[...] coeducación es un proceso educativo, en el que se tienen en cuenta una igualdad entre los dos géneros, que tengan las mismas posibilidades" (\#4). Otro docente añade, "[...] coeducación es trabajar todos los procesos de enseñanza-aprendizaje conjuntamente, sin distinción de sexo, tanto de actividades u otras acciones educativas que se den en este proceso" (\#1). En la misma línea se opina, "[...] para mí, es la igualdad, equidad entre los dos géneros, impartir unos contenidos a los dos géneros, cualquier bloque de contenidos a los dos grupos" (\#7). Por último, se añade que "[...] coeducación es la educación que integra a los dos sexos" (\#9), corroborándolo con la siguiente afirmación "[...] el concepto es amplio, es diferente que convivan en el aula o que se aproveche eso y se le saque partido para alcanzar los objetivos" (\#9). Además, se relaciona con el término inclusión con la siguiente afirmación, "[...] a finales de los 90 comenzó la terminología de educación inclusiva, donde una cosa es que convivan en el centro y otra en que realmente 
este integrados y participen como cualquier otro alumno en las actividades, por lo tanto la coeducación va encaminada al término inclusión" (\#3).

Desde un enfoque más progresista, encontramos que la definición de coeducación se sitúa en una perspectiva de diferencias entre los sexos. Así, Martínez-Galindo (2006) indica que la coeducación trata de hacer participe a los chicos de las formas de conducta que hasta ahora han sido consideradas femeninas. Además, Fontecha (2006) añade que la coeducación es un proceso intencionado de intervención a través del cual se potencia el desarrollo de niñas y niños partiendo de la realidad de dos sexos diferentes. En una de las entrevistas se indica, "[...] lo ideal es que no haya diferencias a la hora de dar clase, puede existir alguna leve diferencia para abordar algunos contenidos. Estas diferencias deben ser usadas para sacar el máximo rendimiento a las actividades de aprendizaje que se puedan realizar" (\#5).

Por último, se tienen en cuenta las ideas que tiene el profesorado sobre modelos de coeducación. En el marco teórico encontramos los modelos de educación en función del género que propone Bonal (1997), el cual nos plantea la educación segregada, mixta y coeducativa. En las entrevistas, las opiniones vertidas son las siguientes:

Un modelo de Coeducación es aquel en el que están en el aula y se les deja que se agrupen espontáneamente según los valores que tienen en la sociedad, televisión, padres, roles que juegan entre ellos. Otro modelo es aquel en el que están interactuando entre grupos que están formados en torno al género. Juegan juntos, pero luego para las agrupaciones buscan los iguales de género (\#8).

Esta opinión gira en torno a una idea de educación mixta en primer lugar, y segregada en segundo lugar, llegando solo a la separación por actividades. Posteriormente, el mismo docente afirmó:

[...]otro modelo está en que provoquemos agrupamientos mixtos en el que interactúen en el mismo grupo chicos y chicas. Lleva mayor problema 


\begin{abstract}
porque es ir contra sus apetencias, y no vale también con que estén hay sino que luego también habrá que diseñar actividades donde tengan algún tipo de interacción, que no sea solo estar juntos por estar juntos, deben prestar atención, crear agrupamientos y concebir el proceso de trabajo entre chicos y chicas no como un producto acabado desde el principio, sino como algo que tiene que ir trabajando en función de los beneficios a obtener o no. Son tipologías diferentes, dentro de los chicos y las chicas hay muchas personalidades, pero si que se va adquiriendo una serie de rasgos y valores comunes (\#8).
\end{abstract}

Esta idea está más en consonancia con el modelo de escuela coeducativo que marca Bonal (1997), ya que se busca un trabajo común de los sexos teniendo en cuenta que tienen diversos intereses.

\title{
3.2 Categoría Utilización del ESPACIO MOTRIZ
}

De acuerdo con Hernández y Rodríguez (2004) y Cantó y Ruiz (2005), se entenderá como espacio motriz aquel que es utilizado durante la participación de la tarea, donde se desarrollan los distintos comportamientos motores y suceden diferentes procesos de socialización. La importancia de esta categoría radica en que de producirse alguna discriminación en la utilización del espacio motriz, éste se convierte en un elemento de análisis y reflexión sobre las creencias y las diferencias de género que se establecen en el mismo (CASTILLO; CORRAL, 2009).

Esta categoría analiza de qué manera el alumnado hace uso del espacio motriz, ya sea una pista polideportiva o un gimnasio en las sesiones de EF o en el patio de recreo. En esta línea, un docente índica, "[...] lo que he observado es que en el patio, los niños están jugando al fútbol y las niñas a juegos de comba(2). En el caso, de que alguna niña juegue al fútbol ya se le estereotipa con características masculinas y viceversa" (\#2)(\#7). De esta manera, se lleva a cabo un tipo de sexismo que se da en función del espacio, tal y como índica Bonal (1997). 
Ya en la clase de EF, se da la siguiente opinión, "[...] los chicos tienden a ocupar el máximo espacio y arrinconar a las chicas. Esto sucede tanto en gimnasios como en espacios abiertos, no obstante, es mucho más evidente en los espacios abiertos" (\#4). De igual manera, queda reflejado en los trabajos de Álvarez-Bueno et al. (1990) y Vázquez et al. (2000). Estos comportamientos los explica García y Asíns (1994), mediante una tendencia cultural de dominación del territorio. González-Boto, Salguero, Tuero y Márquez (2003), por su parte relacionan las pistas deportivas con el desarrollo de contenidos ligados al sexo masculino y las salas para el desarrollo de la expresión corporal, ligada al sexo femenino.

Por otro lado, referente a los contenidos un docente opina que "[...] en función del tipo de contenidos que se desarrollen, un sexo u otro puede llevar el protagonismo de la sesión, haciendo así uso del espacio como lo estimen oportuno" (\#1). Esta afirmación coincide con las conclusiones que Valdivia et al. (2011) exponen en su trabajo.

Por último, y ya en las actividades extraescolares uno de los profesores entrevistados opina que "[...] a pesar de plantear competiciones mixtas, cuando el alumnado se organiza, los chicos se agrupan por un lado y las chicas por otro lado" (\#9).

\subsection{Categoría Agrupaciones}

La importancia de las agrupaciones viene dada por el tipo de relaciones que se establecen dentro del grupo. Entre las diferentes acciones educativas que se pueden llevar a cabo en estas formaciones, se destaca el intercambio de conocimientos, fomento de reflexiones y puesta en común de la colaboración para la adquisición de habilidades.

En esta categoría analizaremos la formación de grupos mediante instancia del profesorado y/o iniciativa de los alumnos, ya sean grupos mixtos o segregados por razón de sexo.

En primer lugar se analizarán la formación de grupos mixtos por imposición del docente. Respecto a ello, se opina que "[...] se suele incitar y motivar a que los grupos sean mixtos, pero en multitud 
de ocasiones se tiene que imponer por norma" (\#5). Otro docente indica que utiliza "[...] los calentamientos con música trabajando por parejas, y es en estos momento cuando percibo que el alumnado tiene mucha vergüenza a agruparse con alguien del otro sexo" (\#3). En cuanto a las dificultades que tiene el profesorado a la hora de formar estos grupos, se opina que "[...] en grupos con multitud de chicas o de chicos se intenta repartir de tal manera que los grupos sean mixtos, no obstante, existen grupos donde es imposible" (\#7). También refiriéndose a las dificultades, "[...] en ocasiones el profesor impone que los grupos sean mixtos, pero no siempre es bien aceptado, por lo que hay que buscar otras estrategias" (\#6), en este sentido Martínez-Galindo (2006) describe que existen actitudes reticentes por parte del alumnado a la formación de grupos mixtos. En cuanto a las estrategias didácticas utilizadas un docente lanza la siguiente opinión, "[...] en expresión corporal, les exijo desde el principio que los grupos sean mixtos, por lo que aunque encontremos barreras, finalmente lo toman por un hábito" (\#2). Esta afirmación, pone en práctica las recomendaciones de Contreras (1998), quien indica que a base de insistir en una determinada formación estructural mixta, estos grupos terminen por convertirse en estables para cualquier tipo de actividad y objetivo.

Por otro lado, se debe analizar si existe la formación de grupos mixtos por iniciativa del alumnado. En este sentido, solo un profesor dio una opinión en esta línea sin detallar como se forman estos grupos.

Es interesante destacar en cuanto a los grupos mixtos se refiere, las chicas se implican menos en las actividades que los chicos, tal y como resaltan en sus investigaciones Koçak, Harris, Kin y Çiçek (2002) y McCarthy, Crawford, Docheff, Myrick y Hussey (1996).

Respecto a la formación de agrupaciones segregadas por razón de sexo, en primer lugar analizaremos aquellas situaciones en las que el alumnado propone este tipo de agrupaciones. En este tipo de agrupaciones Martínez-Galindo (2006) indica que se pone de manifiesto una agresividad masculina, que a veces desemboca en miradas burlonas, comparativas y denigrantes hacia las chicas. En este sentido, un docente indica que "[...] en clase de bachillerato 
cuando propongo algún tipo de juego, se agrupan en función de su género, es algo que me impacta, pues no evolucionan a pesar de que son mayores" (\#4). De esta manera y siguiendo con la edad del alumnado, "[...] si el alumnado es más joven, el caos es mayor si los agrupamientos son libres, ya que no solo se agrupan por el mismo sexo, sino que se agrupan por su amistad, quedando algunos compañeros excluidos" (\#7). En esta línea, se indica que "[...] a la hora de buscar los agrupamientos el papel del profesor es importante en los cursos de primaria y $1^{\circ}$ y $2^{\circ}$ de secundaria, para ayudar a romper ciertos estereotipos" (\#9). Por otro lado, si nos referimos a la actividad se indica "[...] que dependiendo de la actividad física que se realiza, tienden a agruparse por sexos o no" (\#1). Por último, un profesor nos habla de las consecuencias de este comportamiento, opinando que "[...] puede ser conflictivo si algún alumno/a es rechazado pues siempre puede que sus compañeros lo identifiquen con ese rol y no desarrollar relaciones sociales con él" (\#3). De la misma manera, Castillo y Corral (2009), añade que en estos grupos se pone de manifiesto los estereotipos y actitudes que inciden en la formación del esquema corporal del alumnado y su autoconcepto.

Además del tipo de formación de agrupaciones descritas, existen aquellas agrupaciones segregadas por imposición del profesorado. Colwell (1999) defiende que separar a jóvenes por razón de género para realizar actividades puede ser considerado como una discriminación y que no deben ser separados siempre que las diferencias fisiológicas permitan una práctica conjunta. Por su parte, el profesorado defiende estas formaciones ya que "[...] en los ejercicios de fuerza, el trabajo suele ser entre compañeros del mismo género pues normalmente tienen distintos niveles de fuerza y distintas complexiones corporales" (\#5). Por otro lado, se añade que existen "ciertos deportes de contacto en los que para evitar accidentes es conveniente separarlos por sexo, por ejemplo el rugby" (\#7). 


\subsection{Categoría Lenguaje}

Tal y como indica Menéndez (2006), el sexismo lingüístico es el uso discriminatorio del lenguaje por razón de sexo. De esta manera, existe una cierta confusión entre el sexo y el género gramatical.

Dentro de los diferentes factores que se pueden analizar respecto al lenguaje sexista, en esta investigación trataremos el uso consciente o inconsciente por parte del profesorado.

En cuanto al uso inconsciente de lenguaje sexista por parte del profesorado, éste opina que "[...] según mi impresión en mi centro la gran mayoría de gente no es consciente del efecto que tiene su actitud y lenguaje ante la igualdad de las personas por razones de sexo" (\#6). Otro docente va más allá y lo admite como propio, "sin darte cuenta estás nombrando siempre con el género masculino o diseñas actividades enfocadas más hacia un género" (\#2). Esto es debido a que como indican Lomas (2006) y Vázquez et al. (2000), habitualmente el género gramatical y el género sexual coinciden, derivando en un uso habitual del masculino para denominar tanto a hombres como mujeres. No obstante, coincidimos con Castillo et al. (2012), al afirmar que esto supone una discriminación hacia uno de los géneros.

Por otro lado, y en la línea marcada por Álvarez-Bueno et al. (1990), se señala al profesorado masculino como dominador del lenguaje sexista, de esta manera se indica, "[...] creo que entre los hombres hay un trato general simplista hacia la mujer, no se trata de algo despectivo, pero si de ciertos estereotipos" (\#9).

En otro orden de cosas, existe profesorado que opina que no se utiliza lenguaje sexista. "Intentamos que nuestra actuación o lenguaje, no haga sentirse discriminado al alumnado por razón de sexo" (\#3). Por otro lado, otro docente añade que "[...] el lenguaje sexista cada vez ha venido a menos, más aún con la incorporación de profesorado joven" (\#5). 


\subsection{Categoría Materiales}

La importancia del material viene dada por la asociación que puede existir entre ciertos materiales y el sexo. Estos, pueden estar relacionados con determinados estereotipos que pueden generar rechazo en el alumnado (VALDIVIA, 2012).

En esta categoría se analizará la naturaleza que tienen tantos los libros de texto como los materiales propios del área de EF en lo referente a la coeducación.

En cuanto a los libros, el profesorado coincide con Bonal (1997), al afirmar que "[...] por supuesto que hay sexismo y racismo, no hay más que ver las ilustraciones de los libros" (\#7). En la misma línea, otro docente opina que, "[...] hay cosas escandalosas, en cuanto a los simples gráficos, dibujos, actitudes que van mostrando los libros de texto" (\#6). En este sentido, es importante el estudio de González (2005), quien concluye que los contenidos relacionados con el sexo femenino tienen menor presencia en los libros de texto.

En cuanto a los materiales propios de EF, Ariel (2004) indica que son los varones los que sienten mayor curiosidad, buscando el material y manipulándolo. No obstante, "[...] actualmente tenemos más variedad, como balones de diferentes tamaños que se adaptan no solo al sexo sino a la fuerza, ya que las chicas suelen tener menos fuerza y las manos más pequeñas" (\#2). Esta afirmación, sigue la línea descrita por Moreno, Alonso, Martínez-Galindo y Cervelló (2005), quienes indican que una mayor variedad en el material disminuye la carga sexista.

Por otro lado, se coincide con Torres (2005), al afirmase que "[...] con la aparición de nuevos materiales que fomentan los juegos y deportes alternativos, se pueden trabajar contenidos que están exentos de estereotipos sexistas como si puede llevar el fútbol" (\#4). 


\section{APORTACIONES DEL ESTUDIO Y PERSPECTIVAS DE FUTURO}

En función de las categorías analizadas consideramos necesario comprobar si las ideas del profesorado sobre coeducación en las clases de EF se aplican en sus sesiones y son percibidas por el alumnado. De esta manera, se hace esencial que el profesorado tenga una correcta idea sobre que es la coeducación en EF y como se puede y debe trabajarla.

\section{Conclusiones}

Mediante el análisis de contenido de las diferentes entrevistas realizadas, el objetivo planteado en este trabajo se cumple.

De esta manera, en el bloque de contenidos de "Definición de coeducación" se hace patente que no existe unanimidad en el profesorado sobre que es la coeducación y la mayoría piensa que la coeducación es un proceso educativo que debe asegurar la igualdad de trato y la igualdad de acceso a los recursos. Solo un profesor entrevistado tiene en cuenta las diferencias entre sexos para utilizarlas a favor de la educación del alumnado y una mejora de la convivencia educativa.

En la categoría "Utilización del espacio motriz" concluimos que los chicos utilizan la mayoría del espacio y no se desprenden acciones por parte del profesorado que equilibren dicha situación.

Si nos referimos a las "Agrupaciones", en el caso de que sean mixtas dicha organización viene impuesta por el profesorado. El alumnado se agrupa en función de su sexo y la amistad con los compañeros desde secundaria a bachillerato.

Respecto a la categoría "Lenguaje", el profesorado admite que existe un lenguaje sexista en los centros escolares pero que su uso es inconsciente.

En la categoría "Materiales", se percibe sexismo en diferentes aspectos de los libros de texto y se busca un material variado que esté asociado a estereotipos sexistas en las clases de EF. 
En definitiva y respondiendo al objetivo de investigación, el profesorado de EF no tiene una idea formada sobre que significa la coeducación en el área, y en consecuencia, las metodologías utilizadas son limitadas y solamente buscan la igualdad de género, sin utilizar las características de cada sexo como elementos que fomenten la enseñanza y el aprendizaje del alumnado. 
O conceito de coeducação para professores de Educação Física e a metodologia utilizada para seu trabalho.

Resumo: O objetivo desta investigação consistiu em conhecer qual é o conceito geral dos professores de Educação Física (EF) sobre educação mista em EF e que estratégias metodológicas utilizam os professores para trabalhar. Foram realizadas entrevistas semiestruturadas, procedendo-se análise do seu conteúdo. Destaca-se que, na categoria "Definição de coeducação" não existe um consenso entre os professores sobre o que é coeducação. A categoria "Uso de espaço motriz" revela que os meninos usam a maioria do espaço. A categoria "Linguagem" enfatiza que os professores admitem que exista uma linguagem sexista nas escolas, porém o seu uso é inconsciente. Palavras-chave: Educação Física. Metodología. Ensino.

\section{Concept of coeducation in Physical Education teachers and the methodology followed in its practice}

Abstract: The aim of this research was to know the general conception of Physical Education teachers about the coeducation in their own subjects and to find out which methodological strategies are followed by the teachers to work with coeducation. Some semi structured interviews were done to ask for the content analysis. To stand out: in the category "Coeducation definition", there isn't any arrangement by the professorate about what coeducation is. The category "Drive space employment" shows up that the kids use the most part of the space. The category "Language" stands out that the teachers admit that a sexist way of speaking is inadvertently used in the schools by the kids.

Key words: Physical Education. Methodology. Teaching 


\section{REFERÊNCIAS}

ÁLVAREZ-BUENO; G., CANO, S.; FERNÁNDEZ-GARCÍA, E.; LÓPEZ, C., MANZANO, A. Y; VÁZQUEZ, B. Guía para una educación física no sexista. Madrid: Ministerio de Educación y Ciencia, 1990.

ÁLVAREZ-GAYOU, J. Como hacer investigación cualitativa. Fundamentos y metodología. México D.F.: Paidos. 2006.

ANGUERA, M. T. La observación. In: MORENO, C. (Ed.), Evaluación psicológica. Concepto, proceso y aplicación en las áreas de desarrollo y de la inteligencia. Madrid: Sanz y Torres, 2003. p. 271-308.

ARIEL, P. Juntos pero no revueltos: La Educación Física en clave de género. Cuadernos de Pesquisa, São Paulo, v.121, n. 34, p. 59-76, 2004.

AZZARITO, L.; SOLMON, M. An Investigation of Students' Embodied Discourses in Physical Education: A Gender Project. Journal of Teaching in Physical Education, Champaing, II., v. 28, p. 173-191, 2009.

BOLAÑOS, L.M.; JIMÉNEZ, R. La formación del profesorado en género. Revista de Investigación Educativa, Murcia, v. 25, 77-95, 2007.

CAMPOY ARANDA, T. J. Técnicas cualitativas para la educación intercultural. En: PANTOJa VALLEJO, A. ; CAMPOY ARANDA, T. J. (Eds.), Programas de intervención en educación intercultural. Granada: Grupo Editorial Interuniversitario, p. 51-78, 2006.

CANTO, R.; RUIZ, L. M. Comportamiento motor espontáneo en el patio de recreo escolar: análisis de las diferencias por género en la ocupación del espacio durante el recreo escolar. Revista Internacional de Ciencias del Deporte, Madrid, v. 1, n.1, p. 28-45, 2005.

CASTILLO, M. A.; MARTÍNEZ-LÓPEZ, E. J.; ZAGALAZ, M. L. Analysis of the physical education teacher's opinion from the Lakes Region (Chile) about the classes based on a mixed modality. Journal of Sport and Health Research, Jaén, v. 2, n. 2, p.77-94, 2010.

CASTILLO, O. D.; CORRAL, J.A. El profesorado frente a la discriminación de género: uso de la retroalimentación. Cultura y Educación, Madrid, v. 23, n. 4, 487-498, 2011.

CASTILLO, O. D.; ROMERO, S.; GONZÁLEZ, T.; CAMPOS, M. C.. Gender equity in physical education: The use of information. Sex Roles, New York, v. 67, 108-121, 2012.

CERVELLÓ, E.; DEL VILLAR, F.; JIMÉNEZ, R.; RAMOS, L. Y BLÁZQUEZ, F. Clima motivacional en el aula, criterios de éxito de los discentes y percepción de igualdad de trato en función del género en las clases de Educación Física. Enseñanza: Anuario Interuniversitario de Educación, Salamanca, v. 21, p. 379-395, 2003. 
COLWELL, S. Feminisms and Figurational Sociolo Contributions to Understandings of Sports, Physical Education and Sex/Gender. European Physical Education Review, Chester, v. 5, n. 3, p. 219-240, 1999.

CONTRERAS, O. Didáctica de la Educación Física. Un enfoque constructivista. Barcelona: INDE, 1998.

CORBETTA, P. Metodología y técnicas de investigación social. Madrid: Mc Graw Hill, 2007.

FONTECHA, M. Intervención didáctica desde la perspectiva de género en la formación inicial de un grupo de docentes en educación física, Tesis Doctoral, Universidad del País Vasco, Vitoria, 2006.

FREIXAS, A.; GARCÍA, E.; JIMÉNEZ, J. R..; SÁNCHEZ, J. L.; SANTOS, M. A. La coeducación, un compromiso social. Sevilla: Instituto Andaluz de la Mujer, 1993.

GARCÍA, M.; ASINS, C. Cuadernos para la coeducación. La coeducación en Educación Física. Barcelona: Institut de Ciencies de l'Educacio, Universitat Autónoma de Barcelona, 1994.

GONZÁLEZ, M. ¿Tienen sexo los contenidos de Educación Física Escolar? Transmisión de estereotipos de sexo a través de los libros de texto en la etapa de secundaria. Revista Internacional de Medicina y Ciencias de la Actividad Física y del Deporte, Madrid, v. 5, n. 18, p. 77-88, 2005.

GONZÁLEZ-BOTO, R.; SALGUERO, A.;TUERO, C.; MÁRQUEZ, S. La coeducación en Educación Física como reto para superar la discriminación por razón de sexo: Condicionantes históricos e indicadores actuales. In: CONGRESO DE EDUCACIÓN FÍSICA Y DEPORTE ESCOLAR, 5, 2003. ACTAS..., Valladolid: AVAPEF, 2003, p. 391-395.

HEINEMANN, K. Introducción a la Metodología de la Investigación Empírica en las Ciencias del Deporte. Barcelona: Paidotribo, 2008.

HERNÁNDEZ, J.; RODRÍGUEZ, J. P. Los contenidos y objetivos exclusivos del currículo de Educación Física escolar desde la praxiología motriz: los casos de la Educación Secundaria Obligatoria y el Bachillerato en España. Revista de Educación Física, Madrid, n. 90, p. 11-23, 2003.

KOÇAK, S.; HARRIS, M. B.; KIN, A.; ÇIÇEK, S. Physical activity level, sport participation and parental education level in Turkish junior high school students. Pediatric Exercise Science, Champaing, II., v. 14, p. 147-154, 2002.

LILLO, J., BROTONS, P. Y SIMÓN, M. N. Teoría y práctica de la coeducación. Alicante: Diputación Provincial de Alicante, 2006. 
LOMAS, C. En masculino y en femenino argumentos y orientaciones para un uso equitativo del lenguaje. In: VV.AA., Guía de buenas prácticas para favorecer la igualdad entre hombres y mujeres en educación. Sevilla: Consejería de Educación, Junta de Andalucía, 2006. p. 32-42,.

MENÉNDEZ, M. I. Lenguaje Administrativo no sexista. Sevilla: Instituto Andaluz de la Mujer, 2006.

MARTÍNEZ-GALINDO, C. Motivación, coeducación y disciplina en estudiantes de Educación Física, 2006 Tesis (DoctoraDO). Murcia: Universidad de Murcia, 2006.

MCCARTHY, S.; CRAWFORD, S. A.; DOCHEFF, D. M.; MYRICK, L.; HUSSEY, K. Does coeducational physical education help high school students reach their full potential?. Journal of Physical Education, Recreation and Dance, Reston, v. 67, p. 6-8, 1996.

MORENO, J. A.;ALONSO, N.; MARTÍNEZ-GALINDO, C.; CERVELLÓ, E. Motivación, disciplina, coeducación y estado de flow en educación física: Diferencias según la satisfacción, la práctica deportiva y la frecuencia de práctica. Cuadernos de psicología del deporte, Murcia, v. 5, n. 2, p. 225-243, 2005.

PIEDRA, J.; GARcíA-PÉREZ, R.; REBOLLO, M. A.; RIES, F. J. Actitudes hacia la coeducación en Andalucía ¿Es el profesorado de Educación Física diferente?. Revista de Investigación en Educación, Vigo, v. 9, n. 2, 200-208, 2011

RUIZ, U.; CAMPS, A. Investigar los géneros discursivos en el proceso educativo. Revista de Psicodidáctica, Vitoria, v. 14, n. 2, p. 211-228, 2009.

SOLER, S. Actitudes y relaciones de niñas y niños ante contenidos de la educación física en primaria estereotipados por el género: el caso del fútbol. In: VÁZQUEZ, B. (Coord.), Las mujeres jóvenes y las actividades físico deportivas. Madrid: Consejo Superior de Deportes, 2006. p. 119-150,

SUBIRATS, M.; BRULLET, C. Rosa y azul. La transmisión de los géneros en la escuela mixta. Madrid: Instituto de la Mujer. Ministerio de Cultura, 1988.

TORRES, D. Propuesta de Programación Didáctica coeducativa en Educación Física en Educación Primaria: Un caso en la Sierra de Madrid. Retos, Nuevas Tendencias en Educación Física, Deportes y Recreación, Murcia, n. 8, p. 43-49, 2005.

VALDIVIA, P. A. La coeducación en la Educación física escolar. Análisis de las teorías y metodologías asociadas del profesorado y su reflejo en el alumnado. Estudio en la provincia de Jaén, Tesis (Doctorado). Universidad de Jaén, Jaén. 2012.

VALDIVIA, P. A.; SÁNCHEZ, A.; ALONSO, J. I.; ZAGALAZ, M. L. Experiencias coeducativas del profesorado de educación física y relación con los contenidos de la materia. Revista Teoría de la Educación, Salamanca, v. 12, n. 2, p. 300320, 2011. 
VÁZQUEZ, B. La coeducación en Educación Física. Algunas reflexiones. In VVAA (Coords.) Primer postgrado de coeducación. Hacia una escuela coeducadora, Álava: UPV, 1990. p. 291-297.

VÁZQUEZ, B.; FERNÁNDEZ-GARCÍA, E.; FERRO, S. Educación Física y género: Modelos para la observación y el análisis del comportamiento del alumnado y del profesorado. Madrid: Gymnos, 2000.

ZAGALAZ, M. L.; ARTEAGA, M.;CEPERO, M.; MARTOS, M. M.; MORENO, R.; RODRIGO, M. Los temas transversales, interdisciplinariedad y currículum oculto en Educación Física. In: ZAGALAZ, M. L.; CEPERO, M. (Eds.), Educación Física y su Didáctica. Manual para el maestro generalista., Jaén: Jabalcuz, 2000.p. 293318 .

NOTA:

(1)Los relatos del profesorado están numerados de acuerdo con el orden de realización de las entrevistas. Así, el entrevistado número 1 es identificado como $\# 1$, y así sucesivamente.

(2)Juego en el que dos participantes se sitúan a cada extremo de una cuerda larga y comienzan a balancearla de un lado a otro o haciendo círculos completos. Mientras tanto, uno o varios participantes van saltando sin tocar dicha cuerda al ritmo de la canción que escojan.

Dirección Postal:

Pedro Angel Valdivia-Moral

C/Antequera, 75, C. P. 23640

Torredelcampo, Jaén (España)

Recebido em: 08-06-2012

Aprovado em: 04-11-2012 
